$\xi=-1$

\title{
Estimation and application in log-Fréchet regression model using censored data
}

\author{
Hanan H. Alamoudi ${ }^{1,3}$ *, Salwa A. Mousa ${ }^{1,2}$, Lamya A. Baharith ${ }^{1}$ \\ ${ }^{1}$ Department of Statistics, Faculty of Science, King Abdulaziz University, Jeddah, Saudi Arabia \\ ${ }^{2}$ Faculty of Commerce (Girls Campus), Al-Azhar University, Nasr city, Cairo, Egypt \\ ${ }^{3}$ Department of Statistics, Faculty of Science, King Abdulaziz University, P.O.Box 42805, Jeddah 21551, Saudi Arabia \\ *Corresponding author E-mail: hhalamoudi@kau.edu.sa
}

\begin{abstract}
This article introduces a new location-scale regression model based on a log-Fréchet distribution. Maximum likelihood and Jackknife methods are used to estimate the new model parameters for censored data. Martingale and deviance residuals are obtained to check model assumptions, data validity, and detect outliers. Moreover, global influence is used to detect influential observations. Monte Carlo simulation study is provided to compare the performance of the maximum likelihood and jackknife estimators for different sample sizes and censoring percentages. The empirical distribution of the martingale and deviance residuals of the proposed model is examined. A real lifetime heart transplant data is analyzed under the log-Fréchet regression model to illustrate the satisfactory results of the proposed model.
\end{abstract}

Keywords: Fréchet Distribution; Regression Model; Censored Data; Maximum Likelihood; Jackknife Method; Residual Analysis.

\section{Introduction}

The study of the effect of covariate variables on survival time is crucial in many practical applications. Many regression models help to measure this effect. The log-location-scale model is considered as an important type of parametric regression model and commonly used in clinical trials, see Lawless [1]. This model assumes a linear relationship between the $\log$ of lifetime $T$ and the covariate variables.

Several studies were conducted using the log-location-scale regression models. These include the log-Burr XII regression model with censored data analysis by Silva et al. [2], the log-modified Weibull regression models with censored data by Carrasco, Ortega and Paula [3], the log-generalized modified Weibull regression model with censored analysis by Ortega, Cordeiro and Carrasco [4], the log-exponentiated Weibull regression model with intervalcensored analysis by Hashimoto et al. [5], the log-Weibull extended regression model with censored data analysis by Silva, Ortega and Cancho [6], the log-Burr XII regression model with grouped survival data analysis by Hashimoto et al. [7], log-odd log-logistic Weibull regression model with censored data by Cruz, Ortega and Cordeiro [8], log-odd log-logistic generalized half-normal regression model with censored data by Pescim et al. [9].

In this paper, based on log-location-scale regression model and Fréchet distribution, the log-Fréchet (LF) regression model is proposed. Fréchet distribution is considered as type II of the extreme value distribution.There are many applications for extreme value distributions in several fields such as floods, earthquakes, rainfall, sporting, wind speed, queues in supermarkets, and others, see Kotz and Nadarajah [10]. Moreover, the aspects of classical analysis for modeling censored data based on LF regression models are examined. Asymptotic distribution of the maximum likelihood (ML) estimators is carried out which is useful for small sam- ple size since the normality assumptions is not easy to validate. Therefore, the use jackknife estimator is explored for the LF regression model for censored data. Moreover, it is important to examine the assumptions and performs a diagnosis approach after modeling in order to detect influential and outlying observations, see Cook [11].

The article is structured as follows:

Section 2 displays the log-Fréchet distribution. The log-Fréchet regression model is introduced in Section 3. Section 4 presents estimates of model parameters using maximum likelihood and jackknife methods based on censored data. Global sensitivity analysis is discussed in Section 5. In Section 6, residuals analysis is conducted to assess departures from the underlying log-Fréchet model and to detect outliers. Section 7 presents simulation study to estimate model parameters. In Section 8, a medical data set is investigated to show the flexibility and practically of the new regression model. Finally, concluding remarks are presented in Section 9.

\section{The log-Fréchet distribution}

This distribution was introduced by Maurice Fréchet (1878-1973). Assuming that the random variable $T$ follows the standard Fréchet distribution with parameters $\lambda$ and $\gamma$. The cumulative distribution function (CDF) and the corresponding probability density function (PDF) are respectively given by:

$$
\begin{aligned}
& F(t ; \lambda, \gamma)=\exp \left\{-\left(\frac{\gamma}{t}\right)^{\lambda}\right\} t>0, \\
& f(t ; \lambda, \gamma)=\lambda \gamma^{\lambda} t^{-(\lambda+1)} \exp \left\{-\left(\frac{\gamma}{t}\right)^{\lambda}\right\} .
\end{aligned}
$$

Then the random variable $Y=\log (T)$ will have log-Fréchet distribution (LFD) with transformation parameter $\sigma=1 / \lambda$ and $\mu=$ 
$\log (\gamma)$. Therefore, the PDF and CDF for LFD are given as follows:

$F(y ; \sigma, \mu)=\exp \left\{-\exp \left[-\left(\frac{y-\mu}{\sigma}\right)\right]\right\},-\infty<y<\infty$,

$f(y ; \sigma, \mu)=\frac{1}{\sigma} \exp \left\{-\left(\frac{y-\mu}{\sigma}\right)\right\} \exp \left\{-\exp \left[-\left(\frac{y-\mu}{\sigma}\right)\right]\right\}$,

where $\sigma>0$ and $-\infty<\mu<\infty$ are the scale and location parameters respectively. The survival and hazard functions respectively are as follows:

$S(y ; \alpha, \sigma, \mu)=1-\exp \left\{-\exp \left[-\left(\frac{y-\mu}{\sigma}\right)\right]\right\}$,

$h(y ; \alpha, \sigma, \mu)=\frac{\exp \left\{-\left(\frac{y-\mu}{\sigma}\right)-\exp \left[-\left(\frac{y-\mu}{\sigma}\right)\right]\right\}}{\sigma\left[1-\exp \left\{-\exp \left[-\left(\frac{y-\mu}{\sigma}\right)\right]\right\}\right]}$

Fig. 1 shows that the LFD has a single mode. The LFD has a monotonic increasing survival function and a monotonic decreasing hazard function.

For the variable $\mathrm{Y}$, the moment generating function is:

$\mathrm{M}_{\mathrm{Y}}(\mathrm{t})=\exp (\mathrm{t} \mu) \Gamma(1-\mathrm{t} \sigma), \mathrm{t} \sigma<1$

The standardized random variable $Z=(y-\mu) / \sigma$ has density function given by:

$f(z)=\exp \{-z\} \exp \{-\exp [-z]\},-\infty<z<\infty$.

And its survival function is:

$S(z)=1-\exp \{-\exp [-z]\}$.

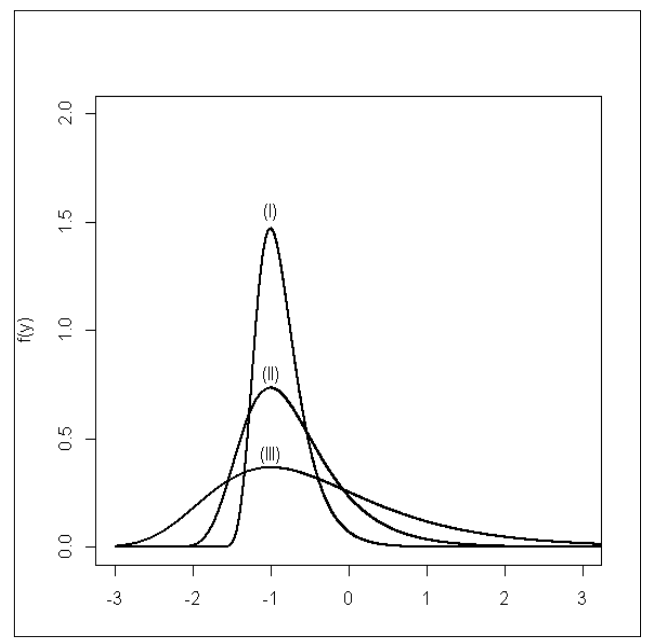

Fig. 1: PDF of the LFD with Parameter $\mu=-1$ and Different Values of Parameter $\sigma=$ (I) 0.25 , (II) 0.5 , And (III) 1 .

\section{The log-Fréchet regression model}

In many real applications for lifetime data, investigation of the relation between the survival time and the independent (explanatory) variables is important. Therefore, the regression model approach can be used. That is, the model in (2) can be written as linear log-location-scale regression model:

$y_{i}=\beta^{T} x_{i}+\sigma z_{i} i=1,2,3, \ldots, n$,

where $z_{i}$ is the random error with density function in (3), $\beta=$ $\left(\beta_{1}, \ldots, \beta_{p}\right)^{T}, \sigma>0$ is a vector of unknown parameters, and $x_{i}=$ $\left(x_{i 1}, \ldots, x_{i p}\right)^{T}$ is the explanatory variable vector. The parameter $\mu_{i}=\beta^{T} x_{i}$ is the location of $Y_{i}$. The location parameter vector $\mu=$ $\left(\mu_{1}, \ldots, \mu_{n}\right)^{T}$ can be represented as a linear model $\mu=\beta^{T} X$, where $X=\left(x_{1}, \ldots, x_{n}\right)^{T}$ is a known model matrix. In this case, the survival function of $\mathrm{Y} \mid \mathrm{x}$ is given by:
$S(y \mid x)=1-\exp \left\{-\exp \left[-\left(\frac{y-\beta^{T} x_{i}}{\sigma}\right)\right]\right\}$.

\section{Estimation}

\subsection{Maximum likelihood estimation}

Let $\left(y_{1}, \tau_{1}, x_{1}\right), \ldots,\left(y_{n}, \tau_{n}, x_{n}\right)$ be a right censored random sample of $n$ observation, where $y_{i}=\left\{\begin{array}{l}\log \left(t_{i}\right) \text { if } \tau_{i}=1 \\ \log \left(c_{i}\right) \text { if } \tau_{i}=0\end{array}, t_{i}\right.$ and $c_{i}$ are lifetimes and censoring times respectively and $x_{i}$ is an explanatory variable. Assuming that the life times and censoring times are random and independent, the log likelihood function is given by:

$$
\begin{aligned}
l(\boldsymbol{\theta}) & =-r \log (\sigma)+\sum_{i=1}^{n} \tau_{i}\left[-z_{i}-\exp \left(-z_{i}\right)\right] \\
& +\sum_{i=1}^{n}\left(1-\tau_{i}\right) \log \left[1-\exp \left[-\exp \left(-z_{i}\right)\right]\right],
\end{aligned}
$$

where $r$ denotes the number of uncensored observations, $\boldsymbol{\theta}=$ $(\sigma, \beta)^{T}$ and $z_{i}=\left(y_{i}-\beta^{T} x_{i}\right) / \sigma$.

By maximizing the log likelihood in (5), the maximum likelihood estimate (MLE) for the parameter vector $\boldsymbol{\theta}$ can be obtained. The nlminb function in the statistical package $R$ is used to obtain the MLE. Also, the covariance estimates for $\widehat{\boldsymbol{\theta}}$ is acquired from the Hessian matrix. Under some regularity conditions, the distribution of $\widehat{\boldsymbol{\theta}}$ is asymptotically normal with covariance matrix that represents the inverse of the Fisher information matrix $I(\boldsymbol{\theta})^{-1}$ where $I(\boldsymbol{\theta})=E\left[-\left(\frac{\partial^{2} l(\boldsymbol{\theta})}{\partial \theta_{j} \partial \theta_{k}}\right)\right]$. However, the presence of censored observations makes the computation of the Fisher information matrix difficult. Therefore, the second derivatives matrix of the loglikelihood can be derived and evaluated at the maximum likelihood estimator $\boldsymbol{\theta}=\widehat{\boldsymbol{\theta}}$. Then the asymptotic normal approximation for $\widehat{\boldsymbol{\theta}}$ could be defined as $\widehat{\boldsymbol{\theta}}^{T} \sim N_{(p+1)}\left\{\boldsymbol{\theta}^{T}, \ddot{L}(\boldsymbol{\theta})^{-1}\right\}$, where $\ddot{L}(\boldsymbol{\theta})=$ $-\left(\frac{\partial^{2} l(\boldsymbol{\theta})}{\partial \theta_{j} \partial \theta_{k}}\right)$ is the $(p+1) \times(p+1)$ observed matrix such that:

$\ddot{L}(\boldsymbol{\theta})=\left(\begin{array}{ll}-\left.\frac{\partial^{2} l(\boldsymbol{\theta})}{\partial \sigma^{2}}\right|_{\widehat{\beta}_{j}, \widehat{\sigma}} & -\left.\frac{\partial^{2} l(\boldsymbol{\theta})}{\partial \sigma \partial \beta_{j}}\right|_{\widehat{\beta}_{j}, \widehat{\sigma}} \\ -\left.\frac{\partial^{2} l(\boldsymbol{\theta})}{\partial \beta_{j} \partial \sigma}\right|_{\widehat{\beta}_{j}, \widehat{\sigma}} & -\left.\frac{\partial^{2} l(\boldsymbol{\theta})}{\partial \beta_{j} \partial \beta_{s}}\right|_{\widehat{\beta}_{j}, \widehat{\beta}_{s}, \widehat{\sigma}}\end{array}\right)$,

where $i, j=1, \ldots, p$, and the submatrices are defined in Appendix A.

\subsection{Jackknife estimation}

Jackknife estimation is used to improve the estimate of the parameter by reducing the bias of the estimate. The method is based on "leave one out" procedure. Miller [12] uses jackknife in linear models to estimate the variance and bias of model parameters, see Tu and Shao [13]. The idea of jackknife method depends on transforming the problem of estimating population parameters into the problem of estimating the mean of the population.

Suppose that $T_{1}, T_{2}, \ldots, T_{n}$ is a random sample and the sample mean $\bar{T}=\sum_{i=1}^{n} \frac{T_{i}}{n}$ is used to estimate population mean. Then the mean sample when $1^{\text {th }}$ observation deleted can be obtained as:

$\bar{T}_{-1}=\frac{\sum_{i=1}^{n} T_{i}-T_{1}}{n-1}$ for which,

$T_{1}=n \widehat{T}-(n-1) \bar{T}_{-1}$

Let $\hat{\theta}$ be the parameter estimator of the whole sample. Then $\hat{\theta}_{-1}$ is the parameter estimate when we drop the $1^{\text {th }}$ observation from the sample. The pseudo-value of the $1^{\text {th }}$ observation can be calculated from (6) as the difference between parameter estimation from whole sample and parameter estimation obtained without the $1^{\text {th }}$ observation. That is: $\tilde{\theta}_{1}=n \hat{\theta}-(n-1) \hat{\theta}_{-1}$ 
The Jackknife estimate of $\theta$, denoted by $\hat{\theta}_{\text {jack }}$ is the average of pseudo-values:

$\hat{\theta}_{\text {jack }}=\frac{1}{n} \sum_{i=1}^{n} \tilde{\theta}_{i}$,

for more details, see Abdi and Williams [14]. Therefore, the jackknife bias estimator is:

$b_{\text {jack }}=(n-1)\left(\hat{\theta}_{\text {jack }}-\hat{\theta}\right)$

An approximate $100(1-\alpha) \%$ confidence interval (CI) for $\theta$ is given by:

$\hat{\theta}_{\text {jack }} \pm t_{\alpha / 2, n-1} s / \sqrt{n}$,

See Sahinler and Topuz [15] and Algamal and Rasheed [16].

\section{Sensitivity analysis}

Sensitivity represents deviation in model output with respect to changes in model's input(s). Global influence is the diagnostic influence depend on case deletion that represent one of the tools to perform sensitivity analysis introduced by Cook [11]. Case deletion is a popular method to investigate the influence of taking out the $i^{t h}$ case from the data on the parameter estimate. This method compare between $\hat{\theta}$ and $\hat{\theta}_{-i}$, where $\hat{\theta}_{-i}$ is MLE when the $i^{\text {th }}$ case is deleted from original data. Then the $i^{\text {th }}$ case could be considered as influential observation if $\hat{\theta}_{-i}$ is far from $\hat{\theta}$.

This methodology was conducted in many statistical models, see for example, Christensen, Pearson and Johnson [17], Davison and Tsai [18], Xie and Wei [19] and Xie and Wei [20]. The case deletion model for the LF regression model (4) is given by:

$Y_{J}=\beta^{\prime} x_{i}+\sigma Z_{i} ; \quad J=1,2,3, \ldots, n, J \neq i$

For model in (7), $\widehat{\boldsymbol{\theta}}_{-i}=\left(\hat{\sigma}_{(i)}, \hat{\beta}_{(i)}\right)^{T}$ denote the ML estimator of $\boldsymbol{\theta}$ when $i^{\text {th }}$ case is deleted. Then the generalized cook distance and likelihood distance are used to measure the effect of the $i^{\text {th }}$ case on the ML estimator $\widehat{\boldsymbol{\theta}}=(\hat{\sigma}, \hat{\beta})^{T}$.

\subsection{Generalized cook distance}

Generalized cook distance is a method that measure global influence defined as the standardized norm of $\hat{\theta}_{-i}-\hat{\theta}$.

$G D_{i}(\theta)=\left(\hat{\theta}_{-i}-\hat{\theta}\right)^{\prime}\{\ddot{L}(\hat{\theta})\}\left(\hat{\theta}_{-i}-\hat{\theta}\right)$, where $\ddot{L}(\hat{\theta})$ is the observed information matrix.

\subsection{Likelihood distance}

The likelihood distance is another method to measure the difference between $\hat{\theta}$ and $\widehat{\theta}_{-i}$.

$L D_{i}(\theta)=2\left\{l(\hat{\theta})-l\left(\hat{\theta}_{-i}\right)\right\}$,

where $l(\hat{\theta})$ is a $\log$ likelihood function of $\theta$ from original data and $l\left(\hat{\theta}_{-i}\right)$ is $\log$ likelihood function of $\theta$ when $i^{\text {th }}$ case is deleted from original data.

\section{Analysis of residual}

Residuals analysis is an important method for checking the appropriateness of the proposed regression model. This will include studying any departure from error assumption and examine any existence of outliers. Several methods for residuals analysis were. introduced in the literature such as Collett [21]. In this study, we will concentrate on the Marginal and deviance methods.

\subsection{Martingale residual}

Martingale residual was proposed by Barlow and Prentice [22]. It is defined as the difference between the observed number of deaths and the expected in the interval $\left(0, t_{i}\right)$ and can be written as: $r_{M_{i}}=\delta_{i}+\log S_{Y}\left(y_{i}, \hat{\theta}\right)$,

where $\delta_{i}$ is the censor indicator that takes 0 if censored and 1 if lifetime and $S_{Y}\left(y_{i}, \hat{\theta}\right)$ is survival function for LF regression model. Then the martingale residual for LF regression model can be written as:

$$
r_{M_{i}}=\left\{\begin{array}{c}
1+\log \left\{1-\exp \left[-\exp \left(-\hat{z}_{i}\right)\right]\right\} \text { if } i \in \text { life time } \\
\log \left\{1-\exp \left[-\exp \left(-\hat{z}_{i}\right)\right]\right\} \text { if } i \in \text { censored }
\end{array},\right.
$$

where $r_{M_{i}}$ takes the range between $-\infty$ and 1 but not symmetrically distributed (skewed). Therefore, transformation of the martingale residual will be used to reduce the skewness.

\subsection{Deviance residual}

Therneau, Grambsch and Fleming [23] introduced the deviance residuals for Cox model with no time-dependent explanatory variables. This residual is more symmetrically about zero from martingale residual and is given by:

$r_{D_{i}}=\operatorname{sign}\left(r_{M_{i}}\right)\left\{-2\left[r_{M_{i}}+\delta_{i} \log \left(\delta_{i}-r_{M_{i}}\right)\right]\right\}^{\frac{1}{2}}$

In parametric regression models, the previous $r_{D_{i}}$ is not a component of the deviance but can be used as a transformation of the martingale residual. Therefore, the deviance residual for LF regression model can be written as:

$r_{D_{i}}=$

$$
\left\{\begin{array}{c}
\operatorname{sign}\left(1+\log \left\{1-\exp \left[-\exp \left(-\hat{z}_{i}\right)\right]\right\}\right) \\
\left\{-2\left[\begin{array}{c}
1+\log \left\{1-\exp \left[-\exp \left(-\hat{z}_{i}\right)\right]\right\}+ \\
\log \left(-\log \left\{1-\exp \left[-\exp \left(-\hat{z}_{i}\right)\right]\right\}\right)
\end{array}\right]\right\}^{\frac{1}{2}} \\
\text { if } i \in \operatorname{life} \operatorname{time} \\
\operatorname{sign}\left(\log \left\{1-\exp \left[-\exp \left(-\hat{z}_{i}\right)\right]\right\}\right) \\
\left\{-2\left[\log \left\{1-\exp \left[-\exp \left(-\hat{z}_{i}\right)\right]\right\}\right]\right\}^{\frac{1}{2}} \\
\text { if } i \in \text { censored }
\end{array}\right.
$$

\section{Simulation study}

Monte Carlo simulation study is conducted to estimate model parameters $\sigma$ and $\beta_{1}$ when $\beta_{0}=2$ is fixed using ML and jackknife methods. This simulation was implemented for different sample sizes, $\mathrm{n}=30,50$, and 100, from Fréchet distribution with the parameters $\lambda=1.5$ and $\gamma=10$ using various percentages of censoring 10, 30 and 50. Table 1 displays ML and jackknife parameters estimates along with the corresponding standard error (SE) for the LF regression model given in (4).

From these results, it can be noted that:

- SE of estimates using ML method is smaller compared to jackknife method.

- As percentage of censoring increased, the SE of parameter estimates increases at the same sample size.

- SE of parameter estimates decreases when the sample size increases.

In addition, simulation study is preformed to examine the form of the empirical distribution of $r_{M_{i}}$ and $r_{D_{i}}$ residuals at the different sample sizes and percentages of censoring. For 1000 samples generated, ML estimates are obtained for the parameters $\sigma, \beta_{1}$ when $\beta_{0}=2$ is fixed and $r_{M_{i}}$ and $r_{D_{i}}$ residuals are calculated. Then a plot of the mean ordered residuals versus the expected quantiles of the standard normal distribution is displayed in Fig. 2 and 3. Appendix B illustrates the algorithm that computes the ML estimates and residuals for LF regression model. 
Table 1: Maximum Likelihood and Jackknife Estimates of the Parameters, SE for the LF Regression Model with $\sigma=1 / 1.5$ and Fixed $\beta_{0}=2$.

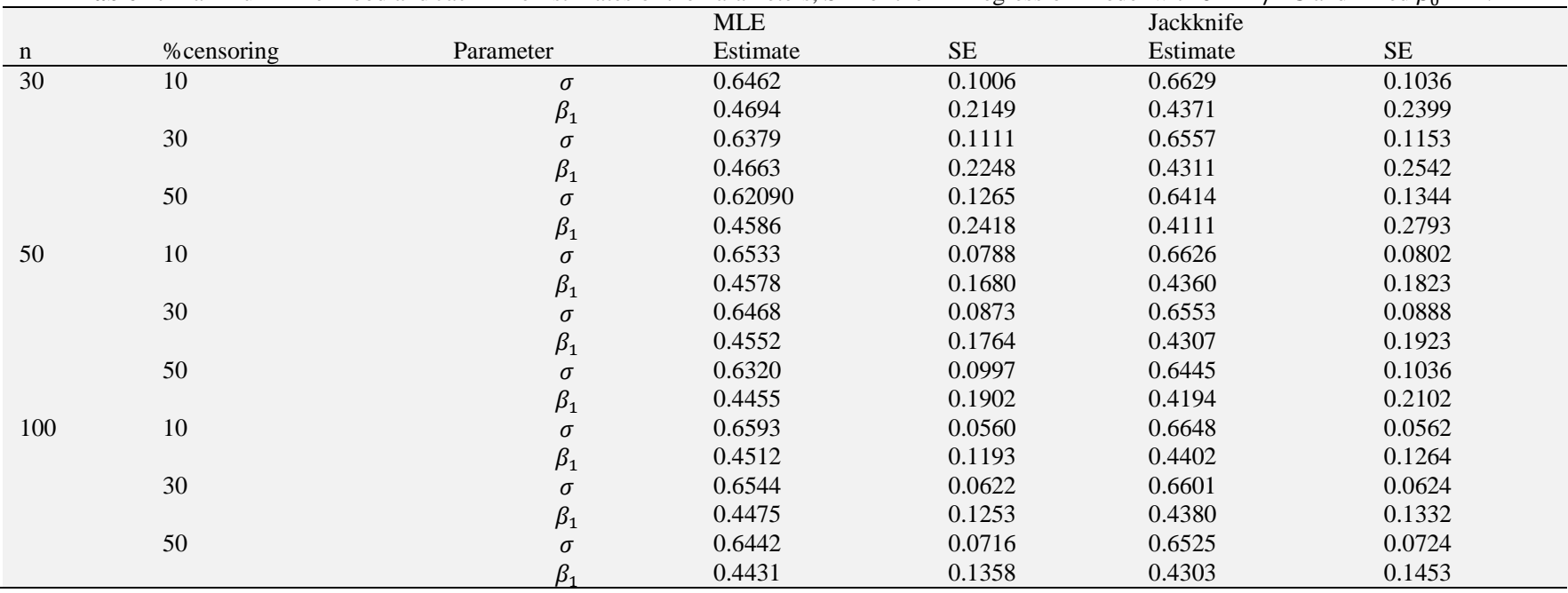

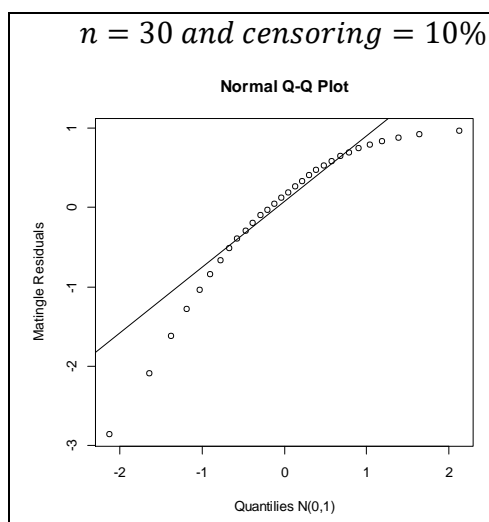

$n=50$ and censoring $=10 \%$

Normal Q-Q Plot

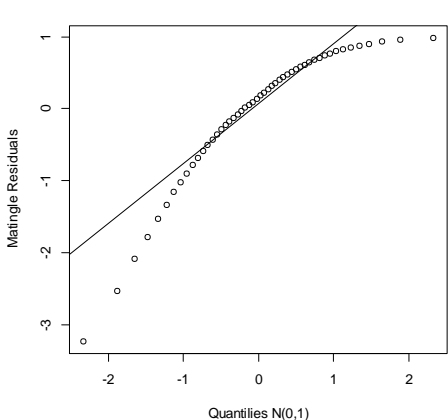

$n=100$ and censoring $=10 \%$

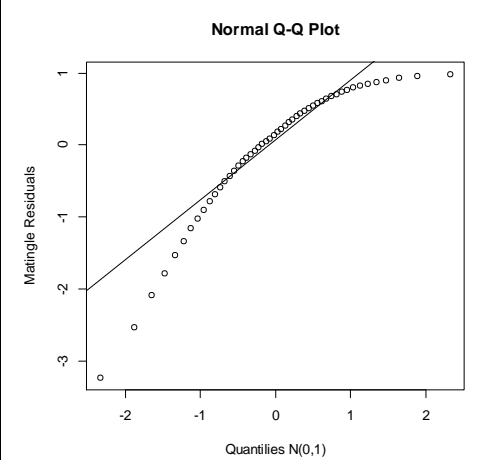

$n=30$ and censoring $=30 \%$

Normal Q-Q Plot

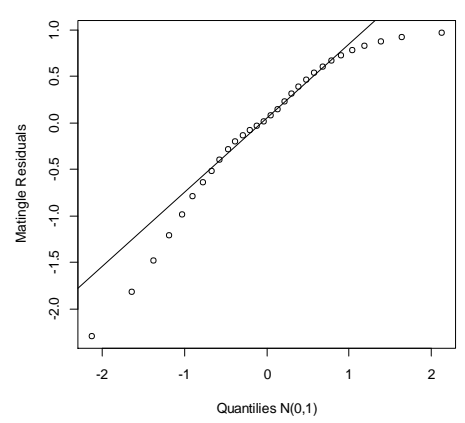

$n=50$ and censoring $=30 \%$

Normal Q-Q Plot

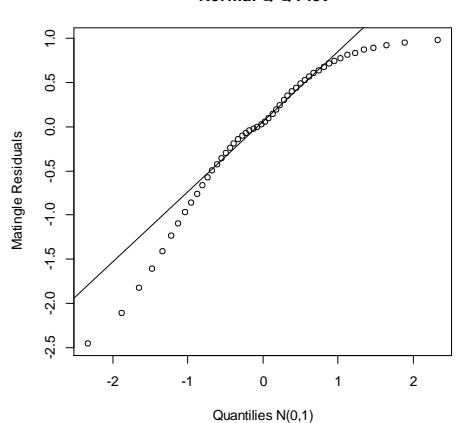

$n=100$ and censoring $=30 \%$

Normal Q-Q Plot

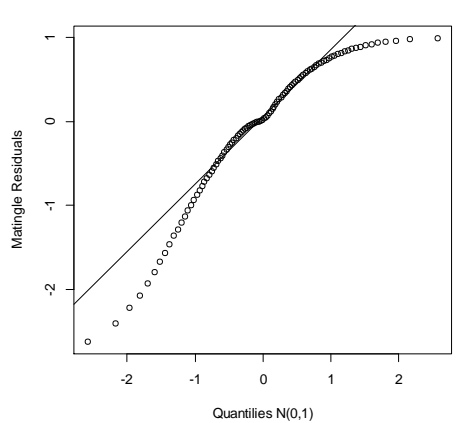

$n=30$ and censoring $=50 \%$

Normal Q-Q Plot

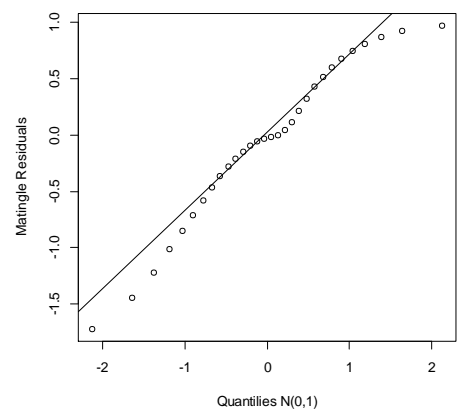

$n=50$ and censoring $=50 \%$

Normal Q-Q Plot

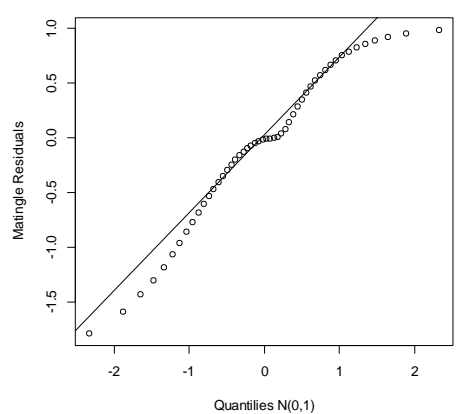

$n=100$ and censoring $=50 \%$

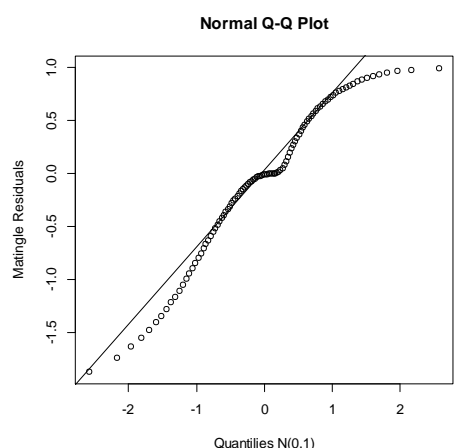

Fig. 2: Normal Probability Plots for the Martingale Residual at Sample Size $n=30,50$ and 100, Different Censoring Percentages $=10,30$ and 50, and Parameter Value $\sigma=1 / 1.5$ and Fixed $\beta_{0}=2$. 


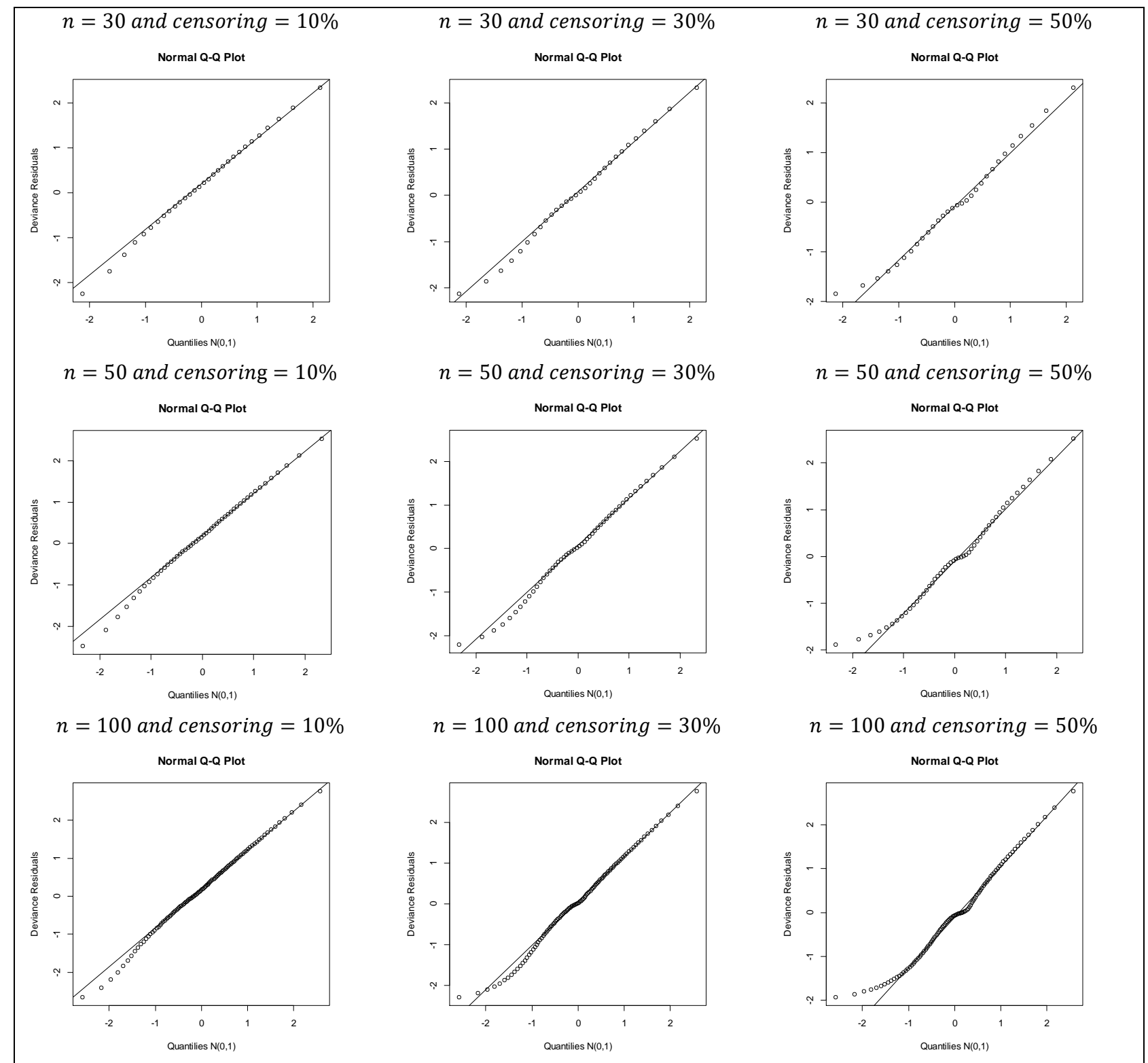

Fig. 3: Normal Probability Plots for the Deviance Residual at Sample Size $n=30,50$ and 100, Different Censoring Percentages $=10,30$ and 50, and Parameter Value $\sigma=1 / 1.5$ and Fixed $\beta_{0}=2$.

From Fig. 2 and 3, we conclude the following:

- The empirical distribution of the deviance residual has close agreement to the standard normal distribution (SND) compared to the martingale residual.

- As the censoring percentage increases, the empirical distribution of the deviance residual moves away from the SND.

- As the sample size increases, the empirical distribution of the deviance residual approaches the SND.

\section{Application}

The Stanford heart transplant data is displayed in Kalbfleisch and Prentice [24] is used to illustrate the performance of the LF regression model. This data represents the survival time of 103 patients since acceptance into transplant program to death. The explanatory variables for each patient consist of age of patient at acceptance and two binary variables prior surgery and transplant.

The following variables in the study are:

$t_{i}$ : Survival time (in days).

$y_{i}: \log$ survival time (in days).

status $_{i}$ : Censoring indicator $(0=$ censoring, $1=$ dead $)$.

$x_{i 1}$ : Age of patients (in years).

$x_{i 2}$ : Previous surgery $(0=$ No, $1=$ Yes $)$. $x_{i 3}$ : Transplant $(0=$ No, $1=$ Yes $)$.

\subsection{Model validation}

To check model validity, a plot of the empirical survival function by Kaplan Meier (KM) estimates and the estimated survival function based on fitting the Fréchet model is shown in Fig. 4. It can be seen from Fig. 4 that the logarithms of the times to event follow the LF distribution.

Therefore, the model fitted can be written as:

$y_{i}=\beta_{0}+\beta_{1} x_{i 1}+\beta_{2} x_{i 2}+\beta_{3} x_{i 3}+\sigma z_{i}, i=1,2, \ldots, 103$,

where $y_{i}$ follows the LF distributions given in (2).

\subsection{Maximum likelihood and Jackknife estimation}

Maximum likelihood and jackknife methods are used to estimate model parameters using nlminb function in $R$ program. SE, $95 \%$ $\mathrm{CI}$ and $\mathrm{p}$-value for each parameter are computed. The results are shown in the Table 2 and 3. It can be observed that the explanatory variables $x_{1}$ and $x_{3}$ are significant for the model at the significance level $5 \%$ for each method but $x_{2}$ is not significant. Also, the estimates from the two methods appear to be very similar. 


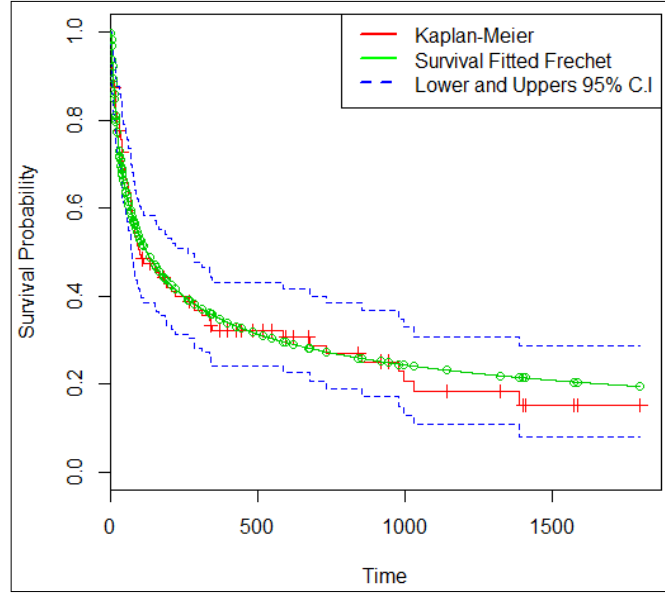

Fig. 4: Plot the Survival Function by Fitting the Fréchet Distribution and KM Function for Leukemia Data.

\subsection{Global influence}

The results of influence measure index plots using heart transplant data for LF regression models are shown in Fig. 5. It is clear that observations 15,38 and 74 are possible influential observations in LF regression model.

\subsection{Analysis of residual}

The deviance residual for the fitted model is represented in Fig.6 (A). It indicates that all observations fall on the interval $(-3,3)$ except observation 38 and are distributed randomly about zero. Therefore, it is expected that observation 38 is outliers.

\subsection{Goodness of fitting}

Fig.6 (B) represents the normal plot for deviance residual with a generated envelope simulation that illustrated in Appendix C. This plot shows that the LF model is suitable to fit the data, since all observations located inside the envelope.

Table 2: The ML Estimates for the Parameters of the LF Regression Model.

\begin{tabular}{cllll}
\hline Parameter & Estimate & SE & $95 \%$ CI & p-value \\
\hline$\sigma$ & 1.7457 & 0.1484 & $(1.4548,2.0366)$ & - \\
$\beta_{0}$ & 4.2129 & 0.9153 & $(2.4189,6.0069)$ & $<0.001$ \\
$\beta_{1}$ & -0.0431 & 0.0189 & $(-0.0801,-0.0061)$ & 0.023 \\
$\beta_{2}$ & 0.6902 & 0.5034 & $(-0.2965,1.6769)$ & 0.170 \\
$\beta_{3}$ & 2.6572 & 0.3782 & $(1.9159,3.3985)$ & $<0.001$ \\
\hline
\end{tabular}

Table 3: The Jackknife Estimates for the Parameters of the LF Regression Model

\begin{tabular}{rllll}
\hline Parameter & Estimate & SE & $95 \%$ CI \\
\hline$\sigma$ & 1.8237 & 0.1839 & $(1.4633,2.1841)$ \\
$\beta_{0}$ & 4.2039 & 0.9893 & $(2.2643,6.1429)$ \\
$\beta_{1}$ & -0.0436 & 0.0211 & $(-0.0850,-0.0022)$ \\
$\beta_{2}$ & 0.5695 & 0.6821 & $(-0.7674,1.9064)$ \\
$\beta_{2}$ & 2.7046 & 0.4555 & $(1.8118,3.5974)$ \\
\hline
\end{tabular}

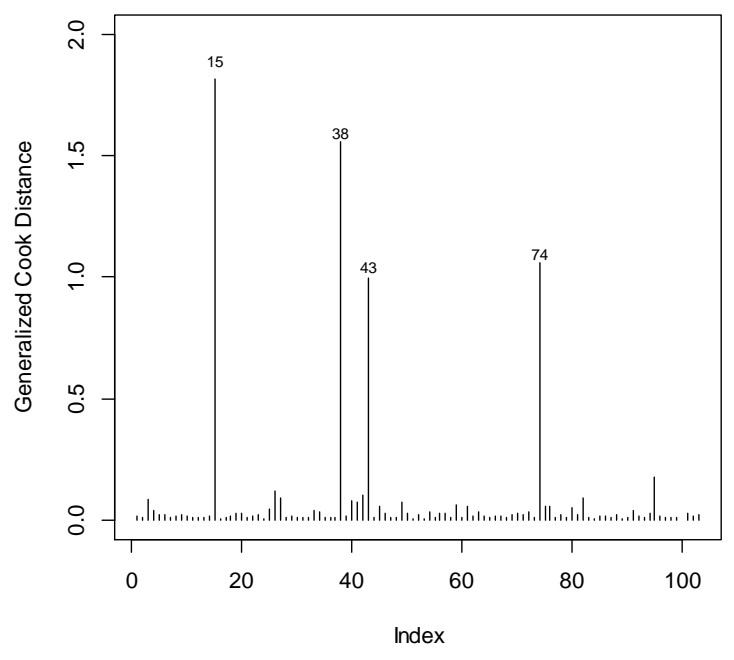

(A)

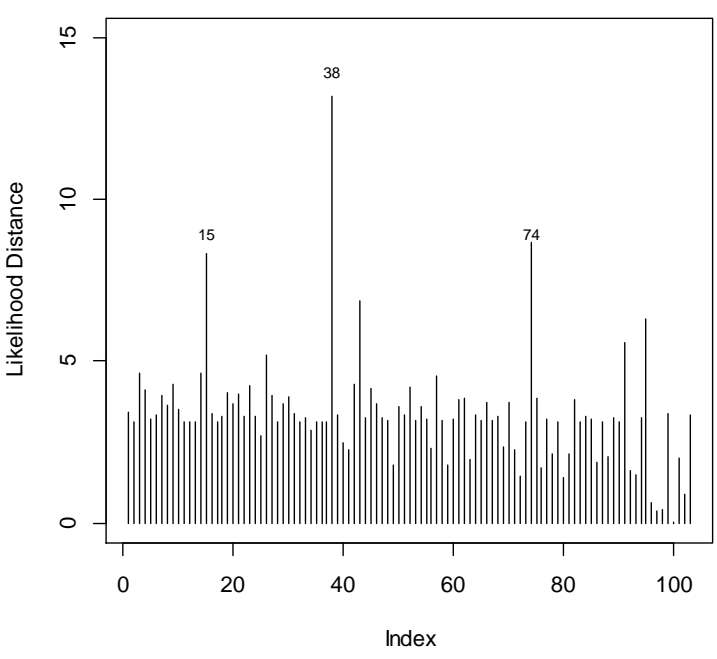

(B)

Fig. 5: Plot Index of (A) Generalized Cook Distance and (B) Likelihood Distance for LF Regression Model. 


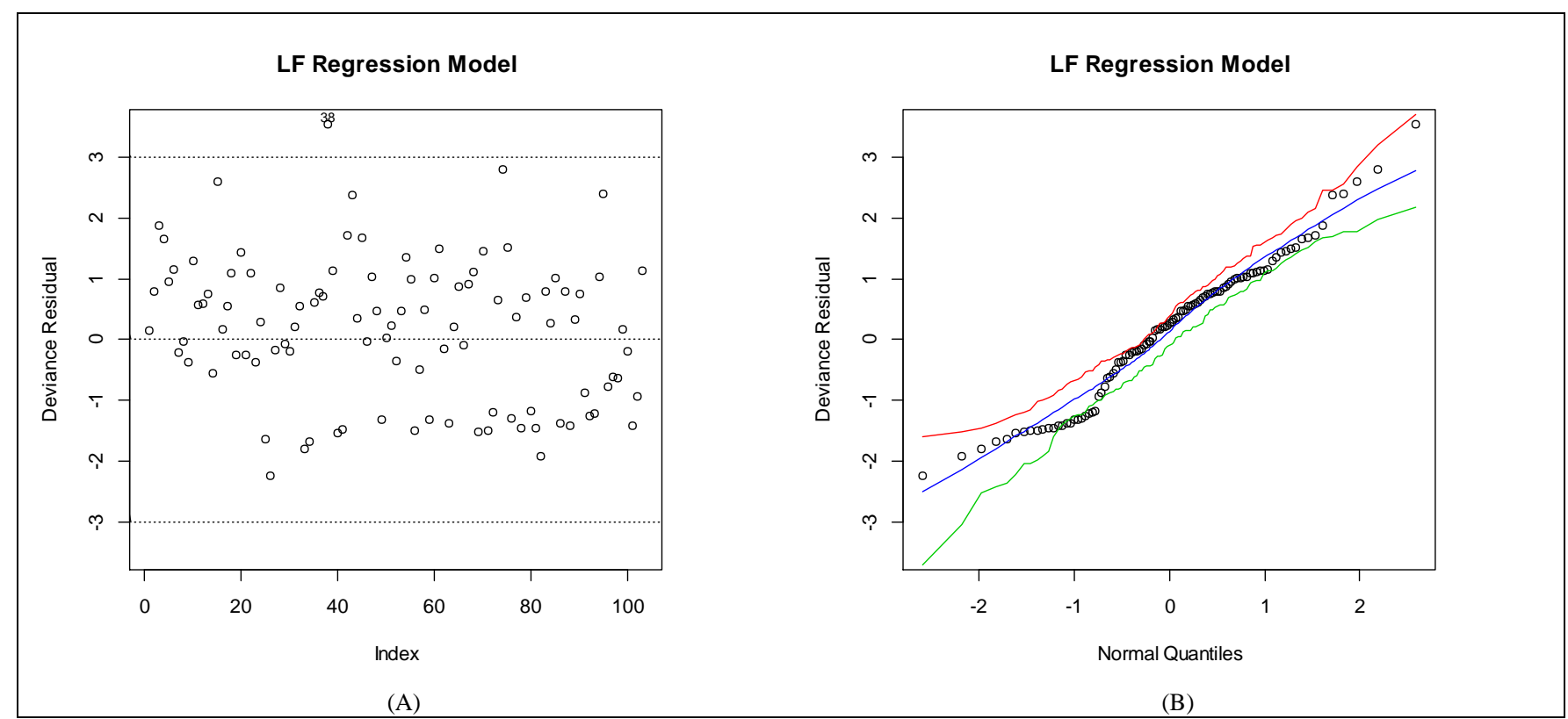

Fig. 6: (A) Index Plot of Deviance Residual. (B) Normal Probability Plot for the Deviance Residual with Envelopes.

\subsection{Comparison between log-weibull and LF regression models}

In Cruz, Ortega and Cordeiro [8], analysis has been conducted on the previous heart transplant survival time data under log-Weibull regression model. Therefore, we conduct a comparison between log-Weibull and LF regression model based on AIC and BIC criteria. Table 4 displays the results of these criteria which show that the LF regression model is more appropriate model compared to the log-Weibull regression model with smaller values of AIC and BIC.

Table 4: Statistics AIC and BIC for Comparing the Log-Weibull and LF Regression Models.

\begin{tabular}{lll}
\hline Model & AIC & BIC \\
\hline Log-Weibull & 353.4208 & 366.5944 \\
LF & 349.1578 & 362.3314 \\
\hline
\end{tabular}

\subsection{Final model}

Based on this analysis, we conclude that the LF regression models are more appropriate for fitting these data compared to logWeibull. Moreover, it can be noted that $\beta_{2}$ is not significant for this model at the level of $5 \%$, see Table 2 . Therefore, the final model fitted is:

$y_{i}=\beta_{0}+\beta_{1} x_{i 1}+\beta_{3} x_{i 3}+\sigma z_{i}, i=1,2, \ldots, 103$,

where $y_{\mathrm{i}}$ follows the LF given in (2).

Table 5 is represents the ML estimates of the parameters in the final model (9). It can be concluded that the log survival time increases for young patients who have received a heart transplant. Fig. 7 represents the survival function corresponding to explanatory variables for the fitted LF regression model.

Table 5: The ML Parameter Estimates of the LF Regression Model - Final Model.

\begin{tabular}{|c|c|c|c|c|}
\hline Parameter & Estimate & SE & p-value & $95 \% \mathrm{CI}$ \\
\hline 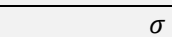 & 1.7492 & 0.1498 & - & $(1.4555,2.04288)$ \\
\hline$\beta_{0}$ & 4.2728 & 0.9029 & $<0.001$ & $(2.50314,6.04239)$ \\
\hline$\beta_{1}$ & -0.0410 & 0.0186 & 0.028 & $(-0.0774,-0.0045)$ \\
\hline$\beta_{3}$ & 2.5562 & 0.3696 & $<0.001$ & $(1.8319,3.2805)$ \\
\hline
\end{tabular}

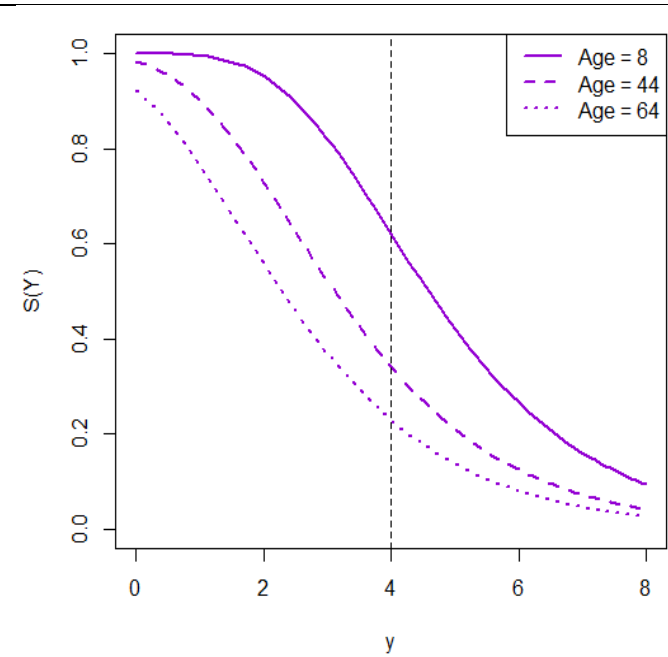

(A)

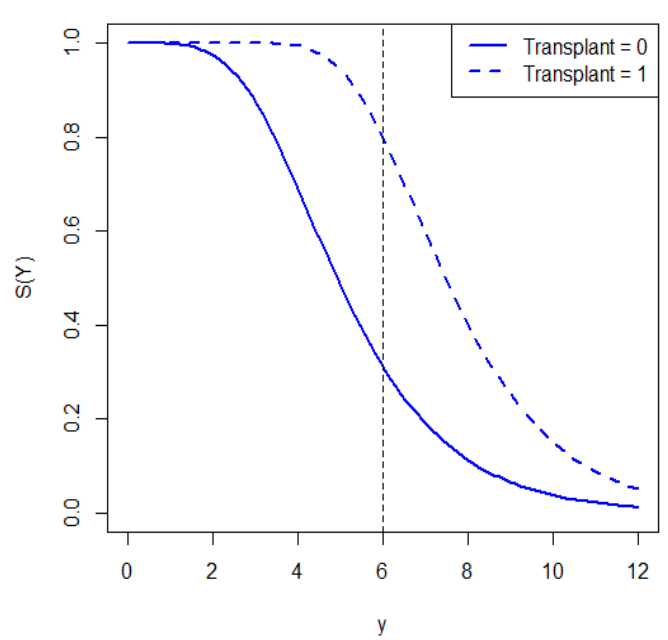

(B)

Fig. 7: Fitted Survival Functions from the LF Regression Model for the Heart Transplant. (A) for $x_{1}=$ Age, (B) for $x_{3}=$ Transplant. 
From Fig. $7(\mathrm{~A})$, it can be seen that $\widehat{\mathrm{S}}(4 \mid$ age $=8)=0.6205$, which means that approximately $62 \%$ of the patients at age equal to 8 years will be alive at $\mathrm{y}=4(\approx 55$ days $)$. Moreover, for patients at age to 44 and $64, \widehat{\mathrm{S}}(4 \mid$ age $=44)=0.3408$ and $\widehat{\mathrm{S}}(4 \mid$ age $=64)=$ 0.2295 , that is, the percentages of the patients that will be alive at $\mathrm{y}=4$ decreased to $34 \%$ and $23 \%$, respectively. Similarly, Fig. $7(B)$, it can be noted that $\widehat{S}(6 \mid$ transplant $=0)=0.3110$, which means that about $31 \%$ of the patients who did not receive a transplant will be alive at $\mathrm{y}=6(\approx 403$ weeks $)$, while for patients who received a transplant, $\widehat{S}(6 \mid$ transplant $=1)=0.7994$, the survival percentage increases approximately to $80 \%$ at $\mathrm{y}=6$.

\section{Concluding remarks}

In this article, a LF regression model with right censored lifetime data is introduced. ML and jackknife methods were used to estimate model parameters. In addition, Monte Carlo simulation is carried out and has indicated that the empirical distribution of the deviance residual approaches the standard normal distribution. Moreover, the robustness features of the ML estimator from the fitted LF regression model are discussed through residuals and sensitivity analysis. The heart transplant data is used to illustrate the performance of the LF regression model. Goodness of fit is conducted for the data by constructing normal probability plot with simulated envelope where we observe that almost all observations fall within the envelope. Finally, the results of analysis showed that the proposed model provided more flexible and appropriate fit for the heart transplant data compared with the logWeibull regression model using AIC and BIC criteria.

\section{References}

[1] J.F. Lawless,Statistical models and methods for lifetime data. 2th Ed, John Wiley and Sons, New Jersey. 2003.

[2] G.O. Silva, E. M. Ortega, V. G. Cancho, M. L. Barreto. Log-Bur XII regression models with censored data. Computational Statistics and Data Analysis 52(7) (2008) 3820-3842. https://doi.org/10.1016/j.csda.2008.01.003.

[3] J.M. Carrasco, E.M. Ortega,G.A. Paula, Log-modified Weibull regression models with censored data: Sensitivity and residual analysis. Computational Statistics and Data Analysis 52(8) (2008) 4021 4039. https://doi.org/10.1016/j.csda.2008.01.027.

[4] E.M. Ortega, G.M. Cordeiro, J.M. Carrasco, The log-generalized modified Weibull regression model. Brazilian Journal of Probability and Statistics0 (00) (2009) 1-29.

[5] E.M. Hashimoto, E.M. Ortega, V.G. Cancho, G.M. Cordeiro, The log-exponentiated Weibull regression model for interval-censored data. Computational Statistics and Data Analysis54 (4) (2009) 1017-1035. https://doi.org/10.1016/j.csda.2009.10.014

[6] G.O. Silva, E.M. Ortega, V.G. Cancho, Log-Weibull extended regression model: estimation, sensitivity and residual analysis. Statistical Methodology7 (6) (2010) 614-631. https://doi.org/10.1016/j.stamet.2010.05.004.

[7] E.M. Hashimoto, E.M. Ortega, G.M. Cordeiro, M.L. Barreto, The Log-Burr XII Regression Model for Grouped Survival Data. Journal of biopharmaceutical statistics 22(1) (2012) 141-159. https://doi.org/10.1080/10543406.2010.509527.

[8] J.N.d.Cruz, E.M. Ortega, G.M. Cordeiro, The log-odd log-logistic Weibull regression model: modelling, estimation, influence diagnostics and residual analysis. Journal of Statistical Computation and $\begin{array}{llll}\text { Simulation } & 86(8) & \text { (2016) }\end{array}$ https://doi.org/10.1080/00949655.2015.1071376.

[9] R.R. Pescim, E.M. Ortega, G.M. Cordeiro, M. Alizadeh, A new log-location regression model: estimation, influence diagnostics and residual analysis. Journal of Applied Statistics (2016) 1-20.

[10] S.Kotz,S. Nadarajah, Extreme value distributions: theory and applications, World Scientific,London, 2000 https://doi.org/10.1142/p191.

[11] R.D. Cook, Detection of influential observation in linear regression Technometrics (1977) 15-18

[12] R.G. Miller, An unbalanced jackknife. The Annals of statistics2 (5) (1974) 880-891. https://doi.org/10.1214/aos/1176342811.
[13] D.Tu, J. Shao, The Jackknife and bootstrap, Springer-Verlag, New York, 1995.

[14] H. Abdi, L. Williams, Jackknife. Encyclopedia of Research Design, Thousand Oaks, CA: Sage, (2010) 1-10.

[15] S. Sahinler,D. Topuz, Bootstrap and jackknife resampling algorithms for estimation of regression parameters. Journal of Applied Quantitative Methods2 (2) (2007) 188-199.

[16] Z.Y. Algamal, K.B. Rasheed, Re-sampling in Linear Regression Model Using Jackknife and Bootstrap. Iraqi Journal of Statistical Science18 (2010) 59-73.

[17] R. Christensen, L.M. Pearson, W. Johnson, Case-deletion diagnostics for mixed models. Technometrics 34(1) (1992) 38-45. https://doi.org/10.2307/1269550.

[18] A.Davison,C.-L. Tsai, Regression model diagnostics. International Statistical Review60 (3) (1992) 337-353 https://doi.org/10.2307/1403682.

[19] F.-C.Xie,B.-C. Wei, Diagnostics analysis for log-BirnbaumSaunders regression models. Computational Statistics and Data $\begin{array}{llll}\text { Analysis51 } & \text { (9) } & \text { (2007a) }\end{array}$ https://doi.org/10.1016/j.csda.2006.08.030.

[20] F.-C. Xie,B.-C. Wei, Diagnostics analysis in censored generalized Poisson regression model. Journal of Statistical Computation and $\begin{array}{llll}\text { Simulation } & 77(8) & \text { (2007b) }\end{array}$ https://doi.org/10.1080/10629360600581316.

[21] D. Collett, Modelling survival data in medical research, CRC press, London, 2003

[22] W.E.Barlow,R.L. Prentice, Residuals for Relative Risk Regression, in Biometrika Biometrika Trust (1988) 65-74.

[23] T.M. Therneau, P.M. Grambsch, T.R. Fleming, Martingale-based residuals for survival models. Biometrika 77(1) (1990) 147-160. https://doi.org/10.1093/biomet/77.1.147.

[24] J.D.Kalbfleisch,R.L. Prentice, The statistical analysis of failure time data. 2thEd, John Wiley \& Sons,New Jersey, 2002. https://doi.org/10.1002/9781118032985.

[25] Y.Zhao, A.H. Lee, K. K. Yau, G. J. McLachlan, Assessing the adequacy of Weibull survival models: a simulated envelope approach. Journal of Applied Statistics 38(10) (2011) 2089-2097. https://doi.org/10.1080/02664763.2010.545115.

\section{Appendix A: Matrix of second derivatives $\ddot{\mathrm{L}}(\theta)$}

$$
\begin{aligned}
& \frac{\partial^{2} l(\theta)}{\partial \sigma^{2}}=\frac{r}{\sigma^{2}}+\sum_{i=1}^{n} \tau_{i}\left[-2 \ddot{z}_{i}-\dot{z}_{i}^{2} \exp \left(-z_{i}\right)+2 \ddot{z}_{i} \exp \left(-z_{i}\right)\right]+ \\
& \sum_{i=1}^{n}\left(1-\tau_{i}\right)\left(\frac{\sigma\left(1-h_{i}\right)\left[-\dot{z}_{i} L_{i}+z_{i} L_{i}\left(\dot{z}_{i}-\dot{z}_{i} \exp \left(-z_{i}\right)\right)\right]-z_{i} L_{i}\left[\left(1-h_{i}\right)+\sigma \dot{z}_{i} L_{i}\right]}{\sigma^{2}\left(1-h_{i}\right)^{2}}\right) \\
& \frac{\partial^{2} l(\theta)}{\partial \beta_{k} \partial \beta_{j}}=\frac{1}{\sigma^{2}} \sum_{i=1}^{n} \tau_{i}\left[-x_{i j} x_{i k} \exp \left(-z_{i}\right)\right]+\frac{1}{\sigma^{2}} \sum_{i=1}^{n}(1- \\
& \left.\tau_{i}\right) x_{i j}\left\{\frac{\left(1-h_{i}\right) L_{i} x_{i k}\left[1-x p\left(-z_{i}\right)\right]-L_{i}{ }^{2} x_{i k}}{\left(1-h_{i}\right)^{2}}\right\}
\end{aligned}
$$

$\frac{\partial^{2} l(\theta)}{\partial \sigma \partial \beta_{j}}=\frac{-1}{\sigma^{2}} \sum_{i=1}^{n} \tau_{i} x_{i j}\left[1-\exp \left(-z_{i}\right)\right]-$

$\frac{1}{\sigma} \sum_{i=1}^{n} \tau_{i} x_{i j} \dot{z}_{i} \exp \left(-z_{i}\right)-\frac{1}{\sigma^{2}} \sum_{i=1}^{n}\left(1-\tau_{i}\right)\left(\frac{x_{i j} L_{i}}{1-h_{i}}\right)$

$+\frac{1}{\sigma} \sum_{i=1}^{n}\left(1-\tau_{i}\right) x_{i j}\left\{\frac{\left(1-h_{i}\right) L_{i} \dot{z}_{i}\left[1-\exp \left(-z_{i}\right)\right]-L_{i}{ }^{2} \dot{z}_{i}}{\left(1-h_{i}\right)^{2}}\right\}$,

where $z_{i}=\left(y_{i}-\beta^{T} x_{i}\right) / \sigma, h_{i}=\exp \left[-\exp \left(-z_{i}\right)\right]$,

$\dot{z}_{i}=\left(y_{i}-\beta^{T} x_{i}\right) / \sigma^{2}, \ddot{z}_{i}=\left(y_{i}-\beta^{T} x_{i}\right) / \sigma^{3}$,

$L_{i}=\exp \left[-z_{i}-\exp \left(-z_{i}\right)\right]$.

\section{Appendix B: Algorithm ML estimation and residual analysis for the parameters of $\mathrm{LF}$ re- gression model}

1) For a given values of the parameters $\lambda=1.5$ and $\gamma=10$, $T_{1}, T_{2}, \ldots, T_{n}$ are lifetimes generated from Fréchet distribution given in (1).

2) Generate the explanatory variable $x_{i}$ from a standard uniform distribution. 
3) Generate $C_{1}, C_{2}, \ldots, C_{n}$ as a censoring time from uniform distribution $[0, \rho]$, where $\rho$ was adjusted until obtaining the required censoring percentages.

4) The $\log$ lifetimes considered in each fit were calculated as $y=\min \left\{\log \left(T_{i}\right), \log \left(C_{i}\right)\right\}$.

5) Estimate model parameter given in (4) by ML method using nlminb function in $R$ program.

6) Compute the standard error for each estimate using hessian function in $R$ program.

7) Compute the standardized, Martingale and deviance residuals.

8) The above steps are repeated 1000 times.

9) Plot the martingale and deviance residuals against the expected quantiles of normal distribution.

\section{Appendix C: Algorithm of envelope simulation for normal probability plots for the deviance residual}

1) Fit the LF regression model (4) to the observed lifetime data.

2) Using the parameter estimates obtained from the fitted model, will generate a sample of $n$ independent observations.

3) Fit the model to generated sample in step (2) based on data set $\left(\tau_{i}, x_{i}\right)$.

4) Compute the values of deviance residuals and ordered them.

5) Repeat steps $(2-4), m$ times.

6) Consider the $n$ sets of the $m$ ordered statistics, compute the mean, minimum, and maximum values across each set.

7) Plot these values and the ordered residuals of the original data against the normal scores.

The minimum and maximum values of the $m$ ordered statistics constitute a simulated envelope to guide assessment of the model adequacy.

See, Ortega, Cordeiro and Carrasco [4] and Zhao et al. [25]. 